\title{
Présentations duales des groupes de tresses de type affine $\tilde{A}$
}

\author{
F. Digne
}

\begin{abstract}
Artin-Tits groups of spherical type have two well-known Garside structures, coming respectively from the divisibility properties of the classical Artin monoid and of the dual monoid. For general Artin-Tits groups, the classical monoids have no such Garside property. In the present paper we define dual monoids for all Artin-Tits groups and we prove that for the type $\tilde{A}_{n}$ we get a (quasi)-Garside structure. Such a structure provides normal forms for the Artin-Tits group elements and allows to solve some questions such as to determine the centralizer of a power of the Coxeter element in the Artin-Tits group.

More precisely, if $W$ is a Coxeter group, one can consider the length $l_{R}$ on $W$ with respect to the generating set $R$ consisting of all reflections. Let $c$ be a Coxeter element in $W$ and let $P_{c}$ be the set of elements $p \in W$ such that $c$ can be written $c=p p^{\prime}$ with $l_{R}(c)=l_{R}(p)+l_{R}\left(p^{\prime}\right)$. We define the monoid $M\left(P_{c}\right)$ to be the monoid generated by a set $\underline{P}_{c}$ in one-to-one correspondence, $p \mapsto p$, with $P_{c}$ with only relations $p p^{\prime}=p \cdot p^{\prime}$ whenever $p, p^{\prime}$ and $p p^{\prime}$ are in $P_{c}$ and $l_{R}\left(p p^{\prime}\right)=l_{R}(p)+l_{R}\left(p^{\prime}\right)$. We conjecture that the group of quotients of $M\left(P_{c}\right)$ is the Artin-Tits group associated to $W$ and that it has a simple presentation (see 1.1 (ii)). These conjectures are known to be true for spherical type Artin-Tits groups. Here we prove them for Artin-Tits groups of type $\tilde{A}$. Moreover, we show that for exactly one choice of the Coxeter element (up to diagram automorphism) we obtain a (quasi-) Garside monoid. The proof makes use of non-crossing paths in an annulus which are the counterpart in this context of the non-crossing partitions used for type $A$.
\end{abstract}

Mathematics Subject Classification (2000). Primary 20F36; Secondary 20 F05.

Keywords. Garside monoid, dual monoid, non-crossing partitions, affine braids.

\section{Introduction}

Soit $(W, S)$ un système de Coxeter quelconque (avec $S$ fini). Soit $R$ l'ensemble des réflexions de $W$ (c'est-à-dire des conjugués des éléments de $S$ ). Appelons "longueur de réflexion" $l_{R}(w)$ d'un élément $w \in W$ le nombre minimum de termes dans une décomposition de $w$ en produits de réflexions. Nous dirons que $v \in W$ divise $w \in W$, noté $v \preccurlyeq w$, si $w=v v^{\prime}$ avec $l_{R}(w)=l_{R}(v)+l_{R}\left(v^{\prime}\right)$. Comme $l_{R}$ est invariant par conjugaison ceci est équivalent à $w=v^{\prime \prime} v$ avec $l_{R}(w)=l_{R}\left(v^{\prime \prime}\right)+l_{R}(v)$, autrement dit il n'y a pas lieu de distinguer entre diviseurs à gauche et à droite. La relation $\preccurlyeq$ 
est clairement une relation d'ordre. Fixons un élément $c$ de Coxeter, c'est-à-dire un produit de tous les éléments de $S$ dans un certain ordre ; considérons l'ensemble $P_{c}$ des diviseurs de $c$ et $M\left(P_{c}\right)$ le monoïde engendré par un ensemble $\underline{P}_{c}=\left\{\underline{w} \mid w \in P_{c}\right\}$ en bijection avec $P_{c}$ avec comme relations $\underline{w} \cdot \underline{w^{\prime}}=\underline{w} w^{\prime}$ si $w$ et $w^{\prime}$ sont des éléments de $P_{c}$ tels que $w w^{\prime} \in P_{c}$ et $l_{R}\left(w w^{\prime}\right)=l_{R}(w)+l_{R}\left(w^{\prime}\right)$ (cf. [BDM, section 2] et [B, 0.2 et 0.4$]$ ). La longueur $l_{R}$ s'étend à $M\left(P_{c}\right)$ en une longueur additive. Les notions de divisibilité à gauche et à droite dans le monoïde étendent la relation de divisibilité de $P_{c}$. Nous conjecturons

Conjecture 1.1. (i) Le groupe des fractions de $M\left(P_{c}\right)$ est isomorphe au groupe des tresses d'Artin-Tits associé à W.

(ii) Le monoüde $M\left(P_{c}\right)$ (resp. son groupe de fractions) a la présentation suivante comme monoïde (resp. comme groupe) : l'ensemble des générateurs est $\{\underline{r} \mid r \in$ $\left.R \cap P_{c}\right\}$ et pour chaque couple $(r, t) \in R^{2}$ tel que $r t$ divise $c$ on a la relation $\underline{r} \cdot \underline{t}=\underline{r} \underline{r} . \underline{.}$.

Cette conjecture a été prouvée pour les groupes de Coxeter de type $A_{n}$ dans [BKL] et $[\mathrm{BDM}]$ et pour les autres types sphériques dans [B]. Dans cet article nous allons prouver cette conjecture dans le cas où $W$ est de type $\tilde{A}_{n}$. Nous montrons de plus que pour exactement un choix $c_{0}$ de l'élément de Coxeter (à automorphisme du diagramme près) le monoïde obtenu est un treillis pour la divisibilité (structure quasi-Garside), ce qui implique l'existence de formes normales dans son groupe de fractions et donne ainsi entre autres conséquences une nouvelle solution au problème des mots dans les groupes d'Artin-Tits de type $\tilde{A}_{n}$. Les preuves utilisent en particulier l'introduction d'objets "sans croisements" dans une couronne, et leur interprétation comme tresses dans un cylindre.

Les symétries des présentations obtenues définissent des automorphismes du groupe d'Artin-Tits dont on peut calculer les points fixes grâce à la théorie des structures de Garside. Cela permet par exemple d'obtenir le centralisateur d'une puissance de l'élément de Coxeter $c_{0}$, qui s'avère être un groupe d'Artin-Tits de type $B$.

Dans la section 2 nous étudions la longueur $l_{R}$ et déterminons $P_{c}$ pour un groupe de Coxeter de type $\tilde{A}$. Nous en donnons une interprétation topologique par des chemins sans croisement dans une couronne. Dans la section 3 nous donnons une présentation du monoïde dual $M\left(P_{c}\right)$. Dans la section 4 nous prouvons la conjecture 1.1 (i) dans

le cas $\tilde{A}$. Dans la section 5 nous prouvons qu'on a une structure de Garside et en donnons un certain nombre de conséquences.

\section{Longueur de réflexion ; diviseurs d'un élément de Coxeter}

On sait par Dyer [D] que la longueur de réflexion d'un élément $w$ d'un groupe de Coxeter quelconque est égale au nombre minimum de termes qu'il faut effacer dans 
une suite minimale d'éléments de $S$ de produit égal à $w$ pour que le produit des termes restants soit égal à 1. C'est aussi la longueur d'un plus court chemin (croissant) de 1 à $w$ dans le graphe de Bruhat du groupe de Coxeter. Ceci montre immédiatement par exemple que la longueur de réflexion d'un élément de Coxeter est égal à sa longueur de Coxeter. Cela montre aussi que la restriction à un sous-groupe parabolique de $l_{R}$ est égale à la longueur de réflexion dans ce sous-groupe. Soit $W$ un groupe de Coxeter de type $\tilde{A}_{n-1}$. Pour étudier $M\left(P_{c}\right)$ nous avons besoin d'une formule explicite pour $l_{R}$ qui nous permettra en particulier de déterminer quels sont les diviseurs d'un élément de Coxeter. Pour cela nous utilisons une représentation de $W$ comme sous-groupe du groupe des permutations de $\mathbb{Z}$. Pour expliquer cette interprétation nous introduisons les définitions suivantes :

Définition 2.1. • Nous dirons qu'une permutation de $\mathbb{Z}$ est $n$-périodique si elle vérifie $w(x+n)=w(x)+n$ pour tout $x \in \mathbb{Z}$.

- Si $w$ est une permutation $n$-périodique de $\mathbb{Z}$, nous appelons décalage de $w$ l'entier $\frac{1}{n} \sum_{x=1}^{x=n}(w(x)-x)$.

On sait ( $c f$. par exemple [S]) que $W$ s'identifie aux permutations $n$-périodiques de $\mathbb{Z}$ de décalage nul. On peut le voir de la façon suivante : le groupe de Coxeter affine de type $\tilde{A}_{n-1}$ est le produit semi-direct du réseau des racines de type $A_{n-1}$ par le groupe de Coxeter de ce type. Autrement dit $W=\mathfrak{S}_{n} \ltimes\left\{\left(a_{i}\right) \in \mathbb{Z}^{n} \mid \sum a_{i}=0\right\}$. Notons $\bar{w}$ l'image dans $\mathfrak{S}_{n}$ de $w \in \mathfrak{S}_{n} \ltimes \mathbb{Z}^{n}$. On peut identifier le groupe $\mathfrak{S}_{n} \ltimes \mathbb{Z}^{n}$ au groupe des permutations $n$-périodiques de $\mathbb{Z}$, l'image de $k \in\{1, \ldots n\}$ par $w=\bar{w} \cdot\left(a_{1}, \ldots, a_{n}\right)$ étant $\bar{w}(k)+n a_{k}$. Alors le décalage d'une permutation $n$-périodique est donné par le morphisme $\bar{w} \cdot\left(a_{1}, \ldots, a_{n}\right) \mapsto a_{1}+\cdots+a_{n}$ et $W$ s'identifie au noyau de ce morphisme.

Si $w$ est une permutation $n$-périodique de $\mathbb{Z}$, l'ensemble des orbites de $w$ est invariant par translation de $n$. Rappelons qu'on appelle support d'une permutation $w$ le complémentaire dans $\mathbb{Z}$ de l'ensemble des points fixes de $w$.

Définition 2.2. Soit $w$ une permutation $n$-périodique de $\mathbb{Z}$; nous appelons décalage d'une orbite $\mathcal{O}$ de $w$ l'entier $\frac{1}{n} \sum_{x \in \mathcal{O} \cap\{1, \ldots, n\}}(w(x)-x)$.

Le décalage de $\mathcal{O}$ est donc l'entier $h$ tel que pour tout $a \in \mathcal{O}$ on ait $w^{k}(a)=a+h n$ où $k$ est le cardinal de l'image de l'orbite modulo $n$. Une orbite est finie si et seulement si elle est de décalage nul. Le décalage d'une permutation $n$-périodique est la somme des décalages de ses orbites, donc une permutation $n$-périodique de $\mathbb{Z}$ est dans $W$ si et seulement si la somme des décalages des orbites infinies est nulle.

On peut décomposer un élément de $W$ en produit d'éléments de $W$ ayant deux à deux des supports disjoints. On s'intéresse aux décompositions maximales de cette forme. Ceci nous amène à poser la définition suivante : 
Définition 2.3. (i) Nous dirons qu'une permutation $n$-périodique est un cycle si elle n'a qu'une orbite non triviale à translation de $n$ près.

(ii) Nous dirons qu'un élément de $W$ différent de l'identité est un pseudo-cycle si la restriction de $w$ à toute partie stricte stable de son support n'est pas dans $W$.

Toute permutation périodique est produit de cycles disjoints et cette décomposition est unique à l'ordre près. Mais un cycle n'est dans $W$ que si son décalage est nul, c'est-à-dire si les orbites de ce cycle sont finies. Tout élément de $W$ est produit de pseudo-cycles de supports disjoints, mais il n'y a pas unicité des pseudo-cycles ayant des orbites infinies (voir par exemple 2.21 ci-dessous). Un pseudo-cycle ou bien est un cycle, ou bien a toutes ses orbites non triviales infinies, de décalage total nul, et dans ce cas toute sous-famille de ses orbites a un décalage total non nul.

Notation 2.4. - Un cycle sera représenté sous la forme $(a, b, c, \ldots, l)_{[h]}$ où $h$ est le décalage du cycle. Cette notation signifiant que $a, b, c, \ldots, l$ sont tous distincts modulo $n$ et que $w$ envoie $a$ sur $b, b$ sur $c, \ldots$ et $l$ sur $a+h n$. Pour simplifier les notations, si le décalage est nul, nous omettrons l'indice [0].

- Nous représenterons toute permutation $n$-périodique de $\mathbb{Z}$ comme un produit de cycles de supports disjoints non vides.

- On pose $s_{i}=(i, i+1)$ pour $i=1, \ldots, n$. Les $s_{i}$ sont les générateurs de Coxeter de $W$.

Avec ces conventions les réflexions sont les éléments $(a, b)$ avec $a$ et $b$ quelconques distincts modulo $n$. On obtient exactement une fois chaque réflexion si on impose de plus $a<b$ et $a \in\{1, \ldots, n\}$.

Nous utiliserons à plusieurs reprises la formule suivante qui résulte d'un calcul immédiat.

Lemme 2.5. On désigne par $a_{1}, \ldots, a_{l}$ des entiers distincts modulo $n$ et par $h$ et $k$ des entiers quelconques. On a

$$
\begin{aligned}
& \left(a_{1}, \ldots, a_{i}, \ldots, a_{l}\right)_{[h]}\left(a_{1}, a_{i}+k n\right) \\
& \quad=\left(a_{i+1}, \ldots, a_{l}, a_{1}+h n\right)_{[h+k]}\left(a_{2}, a_{3}, \ldots, a_{i}\right)_{[-k]} .
\end{aligned}
$$

Définition 2.6. Pour toute partie $T \subset \mathbb{Z}$ invariante par translation de $n$, notons $\tilde{W}_{T}$ le fixateur dans $W$ du complémentaire de $T$ et $W_{T}$ le sous-groupe des éléments de $\tilde{W}_{T}$ n'ayant pas d'orbite infinie. Un sous-groupe du type $W_{T}$ ou $\tilde{W}_{T}$ sera dit "quasiparabolique".

Remarquons que $\tilde{W}_{T}$ et $W_{T}$ sont des groupes de Coxeter de types respectifs $\tilde{A}_{|\bar{T}|-1}$ et $A_{|\bar{T}|-1}$ si $\bar{T}$ est l'image de $T$ modulo $n$. 
Notation 2.7. Pour $w \in W$ notons $v(w)$ le nombre de classes modulo $n$ d'orbites de $w$ et notons $\kappa(w)$ le plus grand entier $k$ tel que $w=w_{1} w_{2} \ldots w_{k}$ où les $w_{i} \in W$ sont (des pseudo-cycles) de supports disjoints.

Remarquons que $\kappa(w)$ est le plus grand entier $k$ tel que $w$ soit dans le produit direct de $k$ sous-groupes quasi-paraboliques. On a $\kappa(w) \leq v(w)$.

La proposition suivante donne une formule explicite pour la longueur de réflexion dans $W$.

Proposition 2.8. Pour $w \in W$ on a $l_{R}(w)=n+v(w)-2 \kappa(w)$.

Preuve. On pose $f(w)=n+v(w)-2 \kappa(w)$. On montre les trois propriétés suivantes :

(1) Si $f(w) \geq 1$, il existe $r \in R$ tel que $f(w r)=f(w)-1$.

(2) Si $f(w)=0$ alors $w=1$.

(3) $f(w) \leq l_{R}(w)$.

Les propriétés (1) et (2) donnent l'inégalité $l_{R}(w) \leq f(w)$, d'où la proposition.

Prouvons (1). Si $w$ a un cycle de la forme $\left(a_{1}, a_{2}, \ldots, a_{l}\right)_{[h]}$ avec $l \neq 1$, la multiplication par $r=\left(a_{1}, a_{2}\right)$, augmente $v(w)$ et $\kappa(w)$ de 1 (cf. 2.5). Si tous les cycles de $w$ sont de la forme $\left(a_{1}\right)_{[h]}$, soit $w_{i}$ un des facteurs différents de l'identité dans la décomposition $w=w_{1} \ldots w_{\kappa(w)}$ comme dans 2.7 ; comme la somme des décalages des orbites de $w_{i}$ est nulle, il est produit d'au moins deux cycles $\left(a_{1}\right)_{[h]}$ et $\left(a_{2}\right)_{[k]}$. Posons $r=\left(a_{1}, a_{2}\right)$; on a $\left(a_{1}\right)_{[h]}\left(a_{2}\right)_{[k]}\left(a_{1}, a_{2}\right)=\left(a_{1}, a_{2}+k n\right)_{[h+k]}$. Donc $v(w r)=v(w)-1$ et $\kappa(w r)=\kappa(w)$, donc $f(w r)=f(w)-1$.

Montrons la propriété (2): Comme $\kappa(w) \leq v(w) \leq n$, si $f(w)$ est nul on doit avoir $v(w)=n=\kappa(w)$. La première égalité prouve que chaque orbite est un singleton modulo $n$ et la deuxième prouve alors que chaque orbite est de décalage nul, donc $w=1$.

Pour montrer la propriété (3) nous montrons que pour $r \in R$ et $w \in W$ on a $f(w r) \leq f(w)+1$. Ceci implique par récurrence que si $w$ est produit de $k$ réflexions on a $f(w) \leq k$.

Soit $r=(a, b)$ une réflexion. Si $a$ et $b$ apparaissent modulo $n$ dans la même orbite de $w$ le lemme 2.5 montre que $v(w r)=v(w)+1$. D'autre part $\kappa(w r) \geq \kappa(w)$, d'où le résultat dans ce cas. Si $a$ et $b$ sont modulo $n$ dans deux orbites différentes du même pseudo-cycle $w_{i}$ de la décomposition $w=w_{1} \ldots w_{\kappa(w)}$, le même lemme montre que $v(w r)=v(w)-1$ et on a $\kappa(w r)=\kappa(w)$. Si $a$ et $b$ apparaissent dans deux pseudocycles distincts, alors de même que précédemment $v(w r)=v(w)-1$ et de plus $w r$ a une décomposition en produit de $\kappa(w)-1$ éléments, donc $\kappa(w r) \geq \kappa(w)-1$, ce qui donne bien $f(w r) \leq f(w)+1$.

Corollaire 2.9. Si w est dans un quasi-parabolique sa longueur de réflexion dans ce sous-groupe est égale à sa longueur de réflexion dans $W$. 
Preuve. On a déjà vu que la restriction de $l_{R}$ à un sous-groupe parabolique est la longueur de réflexion dans ce sous-groupe, grâce au résultat de Dyer. On peut aussi voir que la formule de la proposition 2.8 donne la longueur de réflexion dans un parabolique standard. Ceci donne le résultat dans le cas d'un quasi-parabolique de type $A$. D'autre part, si un élément $w$ est dans le quasi-parabolique de type $\tilde{A}_{n-m-1}$ fixant $j+k n$ pour $j$ dans une partie de cardinal $m$ de $[1, n]$ et pour tout $k$, les valeurs de $v(w)$ et $\kappa(w)$ diminuent de $m$ dans le quasi-parabolique et $n$ est remplacé par $n-m$ dans la formule donnant la longueur. Ceci prouve que $l_{R}$ se restreint bien aussi dans ce cas, d'où le résultat.

Nous allons maintenant chercher quelles réflexions divisent un élément de Coxeter de $W$. La proposition suivante exprime un élément de Coxeter comme permutation.

Proposition 2.10. Soit c un élément de Coxeter de $W$, c'est-à-dire le produit des générateurs de Coxeter dans un ordre arbitraire fixé; alors il existe une partition en deux parties non vides

$$
\{1, \ldots, n\}=\{a, b, \ldots, l\} \coprod\{\alpha, \beta, \ldots, \lambda\}
$$

telle que $a<b<\cdots<$ l et $\alpha<\beta<\cdots<\lambda$ et que

$$
c=(a, b, \ldots, l)_{[1]}(\lambda, \ldots, \beta, \alpha)_{[-1]} .
$$

Preuve. L'ensemble des générateurs de Coxeter est $\left\{s_{1}, s_{2}, \ldots, s_{n}\right\}$ avec les notations de 2.4. On a $c=s_{i_{1}} s_{i_{2}} \ldots s_{i_{n}}$, où les indices $i_{j}$ sont tous distincts. Quitte à changer $c$ en $c^{-1}$, on peut supposer que dans la suite $\left(s_{i_{1}}, s_{i_{2}}, \ldots, s_{i_{n}}\right)$ l'élément $s_{1}$ est à droite de $s_{n}$. Alors $s_{1}$ commute avec tous les $s_{i}$ qui sont à sa droite sauf éventuellement $s_{2}$ qui à son tour commute avec tous les éléments qui sont à sa droite sauf éventuellement $s_{3}$ etc... On peut alors réécrire $c$ comme un produit qui se termine par $s_{1} s_{2} s_{3} \ldots s_{i}$ pour un certain $i$. On itère le procédé en commençant avec $s_{i+1}$. Finalement on écrit $c$ sous la forme

$$
\left(s_{k_{h}} s_{k_{h}+1} \ldots s_{n}\right)\left(s_{k_{h-1}} s_{k_{h-1}+1} \ldots s_{k_{h}-1}\right) \ldots\left(s_{k_{1}} s_{k_{1}+1} \ldots s_{k_{2}-1}\right)\left(s_{1} s_{2} \ldots s_{k_{1}-1}\right),
$$

avec $1<k_{1}<k_{2}<\cdots<k_{h} \leq n$ qui est une permutation de la forme voulue : elle s'écrit $\left(k_{h}, k_{h-1}, \ldots, k_{1}\right)_{[-1]}\left(1,2, \ldots, \widehat{k_{1}} \ldots, \widehat{k_{2}}, \ldots, \widehat{k_{h}}, \ldots, n\right)_{[1]}$, où $\widehat{k_{i}}$ signifie que $k_{i}$ ne figure pas.

Notation 2.11. Dans la suite nous fixons un élément de Coxeter $c$ et posons $X=\{a, b, \ldots, l\}+n \mathbb{Z}$ et $\Xi=\{\alpha, \beta, \ldots, \lambda\}+n \mathbb{Z}$ comme dans la proposition précédente.

Nous allons démontrer : 
Proposition 2.12. Avec les notations ci-dessus, si $x$ et y sont deux entiers distincts, la réflexion $(x, y)$ divise $c$ si et seulement si $x$ et y vérifient l'une des propriétés suivantes:

(i) $x \in X$ et $y \in \Xi$ ou $x \in \Xi$ et $y \in X$.

(ii) $x, y \in X$, et $|y-x|<n$.

(iii) $x, y \in \Xi$ et $|y-x|<n$.

Preuve. On calcule $c(x, y)$ dans tous les cas possibles.

Dans le cas (i), quitte à échanger les rôles de $x$ et $y$ et à changer de représentants des cycles de $c$, on peut supposer $x=l$ et $y=\alpha+n$. Le produit $c(x, y)$ vaut $(a, b, \ldots, l, \lambda, \ldots, \beta, \alpha)$ dont la longueur de réflexion vaut bien $n-1$.

Si $x$ et $y$ sont dans $X$, on peut supposer $x=a$ et on écrit $c=(a, b, \ldots, t, y+$ $k n, z, \ldots, l)_{[1]}(\lambda, \ldots, \beta, \alpha)_{[-1]}$ pour un certain $k$. Le produit $c(x, y)$ vaut $(y, b, \ldots, t)_{[k]}(z, \ldots, l, a+n)_{[1-k]}(\lambda, \ldots, \alpha)_{[-1]}$. Comme $v(c(x, y))=3$, on a $l_{R}(c(x, y))=n-1$ si et seulement si $\kappa(c(x, y))$ est égal à 2 , c'est à dire si on peut regrouper les trois orbites en deux parties de décalages nuls ( $\operatorname{sinon} \kappa(c(x, y))$ est égal à 1$)$. On a donc $\kappa(c(x, y))=2$ si et seulement si $k=0$ ou $k=1$, ce qui donne le cas (ii).

On fait un raisonnement analogue si $x$ et $y$ sont dans $\Xi$ et on obtient le cas (iii).

Les calculs faits dans la preuve précédente montrent aussi que

Corollaire 2.13. Soit s et $t=n-s$ les cardinaux respectifs des images de $X$ et $\Xi$ modulo $n$; un élément de longueur $l_{R}(c)-1$ divise $c$ si et seulement s'il est d'une des trois formes suivantes:

(i) $\left(a_{1}, a_{2}, \ldots, a_{s}, \alpha_{t}, \alpha_{t-1}, \ldots, \alpha_{1}\right)$ où $\left(a_{i}\right)$ et $\left(\alpha_{i}\right)$ sont des sous-suites croissantes formées d'éléments consécutifs respectivement de $X$ et de $\Xi$,

(ii) $\left(a_{1}, a_{2}, \ldots, a_{r}\right)\left(a_{r+1}, \ldots, a_{s}\right)_{[1]}(\lambda, \ldots, \beta, \alpha)_{[-1]}$ avec $\left(a_{i}\right)$ suite croissante d'éléments consécutifs de $X$,

(iii) $(a, b, \ldots, l)_{[1]}\left(\alpha_{t}, \alpha_{t-1}, \ldots, \alpha_{r+1}\right)\left(\alpha_{r}, \alpha_{r-1}, \ldots, \alpha_{1}\right)_{[-1]}$ avec $\left(\alpha_{i}\right)$ suite croissante d'éléments consécutifs de $\Xi$.

Pour donner la liste des diviseurs de $c$ nous avons besoin de la définition suivante.

Définition 2.14. On appelle diviseur élémentaire un pseudo-cycle de $W$ de la forme $\left(a_{1}, \ldots, a_{h}, \alpha_{k}, \ldots, \alpha_{1}\right)$ avec $h \geq 0$, et $k \geq 0$ et $h+k \geq 2$, ou de la forme $\left(a_{1}, a_{2}, \ldots, a_{h}\right)_{[1]}\left(\alpha_{k}, \ldots, \alpha_{2}, \alpha_{1}\right)_{[-1]}$, avec $h \geq 1$ et $k \geq 1$, où les $a_{i}$ sont dans $X$ et les $\alpha_{i}$ dans $\Xi$, et où $a_{1}<a_{2}<a_{3}<\cdots<a_{h}<a_{1}+n$ et $\alpha_{1}<\alpha_{2}<\cdots<$ $\alpha_{k}<\alpha_{1}+n$. 
Remarquons qu'un diviseur élémentaire est un élément de Coxeter d'un quasiparabolique de $W$ : dans le premier cas il s'agit d'un quasi-parabolique de type $A_{h+k-1}$, dans le deuxième d'un quasi-parabolique de type $\tilde{A}_{h+k-1}$.

Nous allons donner une interprétation topologique des diviseurs de $c$ en termes de chemins dans une couronne.

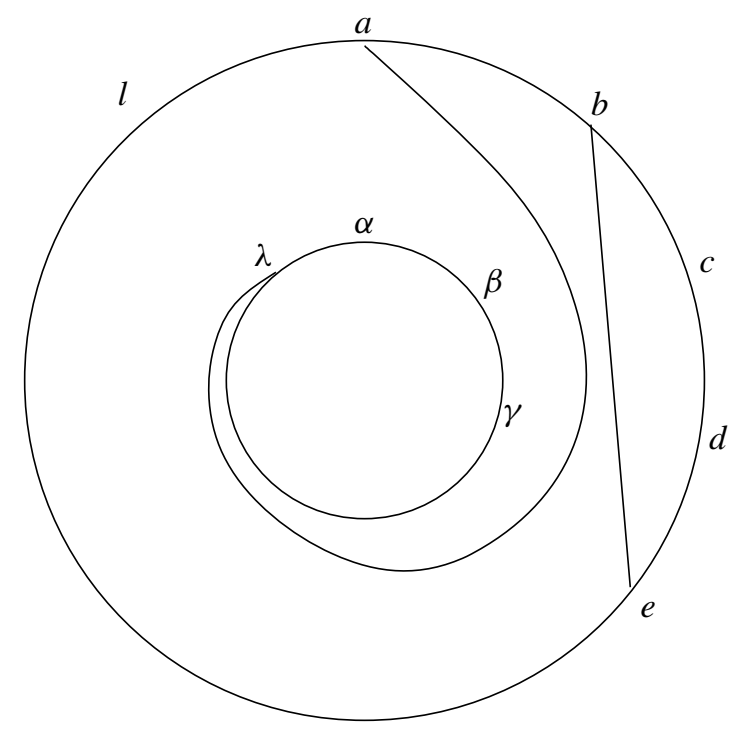

Figure 1. Deux réflexions sans croisement.

On considère une couronne dans le plan orienté. On fixe des points étiquetés $a, b, \ldots, l$ sur le cercle extérieur, dans l'ordre cyclique et on fait de même sur le cercle intérieur avec des points étiquetés $\alpha, \ldots, \lambda$. Cette couronne peut être vue comme le quotient par la translation de $n$ d'une bande infinie orientée où les points de $X$ et de $\Xi$ respectivement sont marqués dans l'ordre croissant sur chacun des deux bords. Nous allons associer à toute réflexion une classe de chemins continus dans la couronne.

Notation 2.15. À la réflexion $(x, y)$ on associe la classe d'homotopie à extrémités fixes de l'image dans la couronne d'un chemin continu joignant $x$ à $y$ dans la bande (voir figure 1, la représentation n'est bien définie qu'une fois fixée l'identification du quotient de la bande par les translations avec la couronne).

Avec cette notation on a :

Corollaire 2.16. Une réflexion divise l'élément de Coxeter c si et seulement si elle peut être représentée par un chemin sans auto-intersection dans la couronne. 
Ce corollaire est une constatation immédiate à partir de la liste donnée dans 2.12.

Pour pouvoir décrire tous les diviseurs d'un élément de Coxeter nous utilisons la même interprétation topologique. À un diviseur élémentaire de la forme $\left(a_{1}, a_{2}, \ldots, a_{h}, \alpha_{k}, \ldots, \alpha_{2}, \alpha_{1}\right)$ nous associons la classe d'homotopie à extrémités fixes du lacet composé des chemins associés aux réflexions $\left(a_{1}, a_{2}\right), \ldots,\left(a_{h-1}, a_{h}\right)$, $\left(a_{h}, \alpha_{k}\right),\left(\alpha_{k}, \alpha_{k-1}\right), \ldots,\left(\alpha_{2}, \alpha_{1}\right),\left(\alpha_{1}, a_{1}\right)$ et à un diviseur élémentaire de la forme $\left(a_{1}, a_{2}, \ldots, a_{h}\right)_{[1]}\left(\alpha_{k}, \ldots, \alpha_{2}, \alpha_{1}\right)_{[-1]}$, nous associons la classe d'homotopie à extrémités fixes de l'union de deux lacets ne se coupant pas et composés respectivement de chemins associés aux réflexions $\left(a_{1}, a_{2}\right), \ldots,\left(a_{h-1}, a_{h}\right),\left(a_{h}, a_{1}+n\right)$ et $\left(\alpha_{k}, \alpha_{k-1}\right)$, $\ldots,\left(\alpha_{2}, \alpha_{1}\right),\left(\alpha_{1}, \alpha_{k}-n\right)$ (voir figure 2$)$.

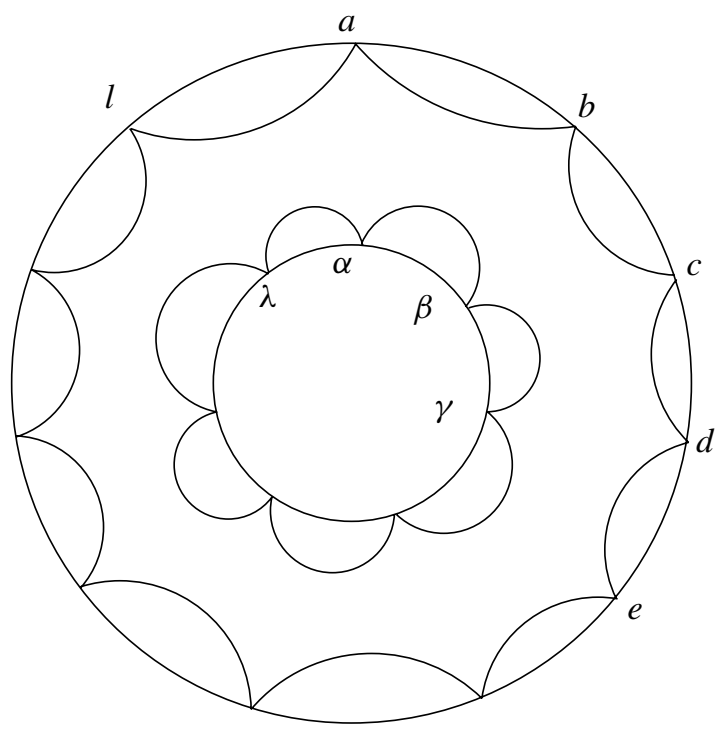

Figure 2. La représentation graphique de $\underline{c}$.

Remarque 2.17. On peut définir de la même façon une interprétation topologique de tout pseudo-cycle. Les diviseurs élémentaires correspondent exactement aux pseudocycles qui ont une représentation (lacet ou union de deux lacets) orientée positivement et sans autointersection.

Définition 2.18. On dit que deux diviseurs élémentaires sont sans croisement s'ils admettent des représentations sans intersection.

En particulier si deux diviseurs élémentaires sont sans croisement, un seul des deux au plus a des orbites infinies car deux lacets d'image non triviale dans le groupe 
fondamental de la couronne et ayant tous deux leur origine sur la même composante du bord ont nécessairement une intersection.

Avec cette définition on peut caractériser les diviseurs d'un élément de Coxeter :

Proposition 2.19. Les diviseurs de l'élément de Coxeter c sont exactement les produits de diviseurs élémentaires deux à deux sans croisement. La longueur d'un tel produit est la somme des longueurs des facteurs et ces facteurs commutent deux à deux.

La remarque qui précède la proposition implique alors qu'un diviseur de $c$ a zéro ou deux orbites infinies (et celles-ci, si elles existent, sont l'une de décalage 1, l'autre de décalage -1).

Preuve. Montrons d'abord l'additivité des longueurs. Dans la formule donnant la longueur, seul le terme $\kappa$ n'est pas toujours additif. Mais pour un élément qui a 0 ou 2 orbites infinies la décomposition en pseudo-cycles est unique, un des pseudo-cycles étant formé de la permutation induite sur l'union des deux orbites infinies. Dans ce cas $\kappa$ est bien additif.

Pour prouver l'assertion sur la forme des diviseurs de $c$ nous utilisons le lemme suivant :

Lemme 2.20. Soient $x$ et $y$ deux entiers distincts et $v$ un élément de $W$ ayant 0 ou 2 orbites infinies.

(i) La réflexion $(x, y)$ divise $v$ si et seulement si elle divise un des pseudo-cycles de $v$.

(ii) La réflexion $(x, y)$ divise un pseudo-cycle si et seulement si $x$ et $y$ sont dans la même orbite de ce pseudo-cycle ou si chacun d'eux est dans une des orbites infinies de ce pseudo-cycle.

Preuve. Si $x$ est dans une orbite finie de $v$ et $y$ dans une orbite finie ou infinie distincte de celle de $x$, alors le calcul de 2.5, appliqué avec $a_{1}=y, a_{i}=x$ et $k=0$, et en faisant passer $(x, y)$ dans le membre de droite, montre que $v(v(x, y))=v(v)-1$ et que la suite des décalages non nuls des orbites est la même pour $v$ et $v(x, y)$. Donc $\kappa(v(x, y))=\kappa(v)-1$ et $l_{R}(v(x, y))=l_{R}(v)+1$, donc $(x, y)$ ne divise pas $v$. Réciproquement, si $x$ et $y$ sont dans le support d'un même pseudo-cycle de $v, 2.5$ appliqué avec $a_{1}=x, a_{2}=y$ et $h=0$ et avec ou bien $k=1$ ou bien $k=0$ montre que $(x, y)$ divise ce pseudo-cycle. Comme les longueurs des pseudo-cycles de $v$ s'ajoutent d'après le début de la démonstration de la proposition, on en déduit que $(x, y)$ divise $v$.

Nous prouvons maintenant, par récurrence descendante sur la longueur du diviseur, l'assertion de la proposition 2.19 sur les diviseurs de $c$. Il y a exactement un 
diviseur de longueur $n$ qui est $c$ lui-même. Un diviseur de longueur $k$ est le produit d'un diviseur $v$ de longueur $k+1$ par une réflexion $(x, y)$ qui divise $v$. Par hypothèse de récurrence appliquée à $v$, les pseudo-cycles de $v$ sont des diviseurs élémentaires deux à deux sans croisement. D'après le lemme précédent la réflexion $(x, y)$ divise $v$ si et seulement si elle divise un des pseudo-cycles $v_{1}$ de $v$. On est ramené à trouver tous les diviseurs de longueur $l_{R}\left(v_{1}\right)-1$ d'un diviseur élémentaire $v_{1}$. Si $v_{1}$ a deux orbites infinies, c'est un élément analogue à $c$ dans le sous-groupe quasi-parabolique correspondant au support de $v_{1}$. Les diviseurs cherchés sont donnés par 2.13. Ce sont bien des produits de diviseurs élémentaires deux à deux sans croisement, de supports inclus dans le support de $v_{1}$, donc aussi sans croisement avec les autres pseudo-cycles de $v$. Si $v_{1}$ n'a pas d'orbite infinie il s'écrit $\left(a_{1}, \ldots, a_{h}\right)$ où $l_{R}\left(v_{1}\right)=h-1$ et ses diviseurs de longueur $h-2$ sont exactement les éléments $\left(a_{1}, a_{2}, a_{i-1}, a_{j+1}, \ldots, a_{h}\right)\left(a_{i}, a_{i+1}, a_{i+2}, \ldots, a_{j}\right)$, ce qui est aussi de la forme annoncée.

Réciproquement, si $v$ est un produit de diviseurs élémentaires deux à deux sans croisement et si $l_{R}(v)<n$ on va montrer qu'on peut trouver une réflexion $r$ telle que $r v$ soit de longueur $l_{R}(v)+1$ et soit un produit de diviseurs élémentaires deux à deux sans croisement. L'hypothèse de récurrence montre alors que $r v$ divise $c$, donc que $v$ divise $c$. Distinguons plusieurs cas pour $v$.

- Si $v$ a une orbite infinie, il en a alors exactement deux, d'après la remarque qui suit 2.18 et ces orbites sont de décalages 1 et -1 , donc les cycles correspondants sont de la forme $\left(a_{1}, a_{2}, \ldots, a_{h}\right)_{[1]}$ et $\left(\alpha_{k}, \ldots, \alpha_{2}, \alpha_{1}\right)_{[-1]}$ avec $a_{1}<a_{2}<a_{3}<$ $\cdots<a_{h}<a_{1}+n$ et $\alpha_{1}<\alpha_{2}<\cdots<\alpha_{k}<\alpha_{1}+n$, les $a_{i}$ étant dans $X$ et les $\alpha_{i}$ dans $\Xi$; d'autre part $v$ doit avoir aussi une orbite finie car $v \neq c$. Tous les points d'une telle orbite finie sont compris entre deux éléments consécutifs d'une des deux orbites infinies, puisque les diviseurs élémentaires sont sans croisement. On peut supposer que cette orbite est comprise dans $\left[a_{1}, a_{2}\right]$. Notons-la $\left\{b_{1}, \ldots, b_{l}\right\}$ avec $a_{1}<b_{1}<b_{2}<\cdots<b_{l}<a_{2}$. On a alors

$$
\begin{aligned}
\left(a_{2}, b_{1}\right) & \left(\alpha_{k}, \ldots, \alpha_{2}, \alpha_{1}\right)_{[-1]}\left(a_{1}, a_{2}, \ldots, a_{h}\right)_{[1]}\left(b_{1}, \ldots, b_{l}\right) \\
& =\left(\alpha_{k}, \ldots, \alpha_{2}, \alpha_{1}\right)_{[-1]}\left(a_{1}, b_{1}, \ldots, b_{l}, a_{2}, \ldots, a_{h}\right)_{[1]} .
\end{aligned}
$$

Si $v$ n'a pas d'orbite infinie, il a au moins une orbite finie de la forme $\left(a_{1}, a_{2} \ldots, a_{h}\right.$, $\left.\alpha_{k}, \ldots, \alpha_{2}, \alpha_{1}\right)$ avec les mêmes conventions que précédemment (cette orbite peut être un singleton). Quitte à échanger les rôles de $X$ et de $\Xi$ on peut supposer que $h \neq 0$. Soit $b_{1}$ le successeur de $a_{h}$ dans $X$. Il y a trois cas :

- Si $b_{1}=a_{1}+n$ alors

$$
\begin{aligned}
\left(b_{1}, \alpha_{k}\right) & \left(a_{1}, a_{2} \ldots, a_{h}, \alpha_{k}, \ldots, \alpha_{2}, \alpha_{1}\right) \\
& =\left(a_{1}, a_{2}, \ldots, a_{h}\right)_{[1]}\left(\alpha_{k}, \ldots, \alpha_{2}, \alpha_{1}\right)_{[-1]} .
\end{aligned}
$$


- Si $k \neq 0$ et si le cycle de $v$ dont le support contient $b_{1}$ est de la forme $\left(b_{1}, b_{2} \ldots, b_{l}, \beta_{m}, \ldots, \beta_{2}, \beta_{1}\right)$ avec $b_{1}<b_{2}<\cdots<b_{l}<b_{1}+n$ et $\beta_{1}<$ $\beta_{2}<\cdots<\beta_{m}<\beta_{1}+n$, les $b_{i}$ étant dans $X$ et les $\beta_{i}$ dans $\Xi$; alors

$$
\begin{aligned}
\left(b_{1}, \alpha_{k}\right) & \left(a_{1}, a_{2}, \ldots, a_{h}, \alpha_{k}, \ldots, \alpha_{2}, \alpha_{1}\right)\left(b_{1}, b_{2}, \ldots, b_{l}, \beta_{m}, \ldots, \beta_{2}, \beta_{1}\right) \\
= & \left(a_{1}, a_{2}, \ldots, a_{h}, b_{1}, b_{2}, \ldots, b_{l}, \beta_{m}, \ldots, \beta_{2}, \beta_{1}, \alpha_{k}, \ldots, \alpha_{2}, \alpha_{1}\right) .
\end{aligned}
$$

- Si $k=0$ et si le cycle de $v$ dont le support contient de $b_{1}$ est de la forme

$$
\left(b_{1}, b_{2} \ldots, b_{r}, \beta_{m}, \ldots, \beta_{2}, \beta_{1}, b_{r+1}, \ldots, b_{l}\right)
$$

avec $b_{r+1}<b_{r+2}<\cdots<b_{l}<a_{1}<a_{h}<b_{1}<b_{2}<\cdots<b_{r}<b_{r+1}+n$ et $\beta_{1}<\beta_{2}<\cdots<\beta_{m}<\beta_{1}+n$, les $b_{i}$ étant dans $X$ et les $\beta_{i}$ dans $\Xi$; alors

$$
\begin{aligned}
\left(a_{1}, b_{1}\right) & \left(a_{1}, a_{2}, \ldots, a_{h}\right)\left(b_{1}, b_{2} \ldots, b_{r}, \beta_{m}, \ldots, \beta_{2}, \beta_{1}, b_{r+1}, \ldots, b_{l}\right) \\
& =\left(a_{1}, a_{2}, \ldots, a_{h}, b_{1}, b_{2}, \ldots, b_{r}, \beta_{m}, \ldots, \beta_{2}, \beta_{1}, b_{r+1}, \ldots, b_{l}\right) .
\end{aligned}
$$

Dans tous les cas le produit est bien comme annoncé.

Enfin puisque les longueurs de diviseurs élémentaires deux à deux sans croisements s'ajoutent et que de tels diviseurs commutent dans $W$, ils commutent aussi dans le monoïde.

Remarque 2.21. Il est faux que la longueur d'un produit de pseudo-cycles de supports disjoints est la somme des longueurs des pseudo-cycles, comme le montre l'exemple suivant dans $\tilde{A}_{n}$ avec $n \geq 5$ :

$$
w=\left[(1)_{[-1]}(2)_{[-1]}(3)_{[2]}\right]\left[(4)_{[1]}(5)_{[1]}(6)_{[-2]}\right] .
$$

La longueur de chacun des facteurs est égale à $4(v=3, \kappa=1$, dans un quasiparabolique de type $\tilde{A}_{2}$ ) et la longueur du produit vaut 6 car le même élément s'écrit

$$
w=\left[(1)_{[-1]}(4)_{[1]}\right]\left[(2)_{[-1]}(5)_{[1]}\right]\left[(3)_{[2]}(6)_{[-2]}\right],
$$

ce qui prouve que $\kappa$ vaut 3 (et on a $v=6$ ) dans un quasi-parabolique de type $\tilde{A}_{5}$.

Par contre s'il y a au plus deux orbites infinies on a bien additivité des longueurs.

À tout diviseur $w$ de $c$ on peut associer la partition périodique de $\mathbb{Z}$ dont les parties sont les supports des pseudo-cycles de $w$.

Nous dirons que deux parties $A$ et $B$ de $\mathbb{Z}$ sont sans croisement si pour tous $x$ et $y$ dans $A$ et tous $z$ et $t$ dans $B$ il existe deux chemins sans intersection dans la bande joignant respectivement $x$ à $y$ et $z$ à $t$. On peut alors réexprimer 2.19 et 2.17 par :

Corollaire 2.22. Une partition périodique de $\mathbb{Z}$ dont toute partie infinie rencontre à la fois $X$ et $\Xi$ est associée comme ci-dessus à un diviseur (unique) de c si et seulement si ses parties sont deux à deux sans croisement. 


\section{Les monoïdes duaux}

L'objectif de cette partie est de prouver la conjecture 1.1 (ii) pour le type $\tilde{A}$. Nous suivons une démarche analogue à celle de $[\mathrm{B}]$ (ou de $[\mathrm{BDM}]$ ). En particulier nous nous plaçons dans le cadre des groupes positivement engendrés telle qu'elle est exposée dans [B, 0.4 et 0.5$]$. Rappelons-en les résultats principaux dans un cadre plus général car nous ne supposons pas que le nombre de générateurs est fini. Soit $G$ un groupe engendré comme monoïde par un ensemble $R$. On dit que $(G, R)$ est un groupe positivement engendré. On définit la longueur dans $l_{R}(g)$ par rapport à $R$ de $g \in G$ comme le nombre minimum de facteurs dans une décomposition de $g$ en produit de générateurs. On dit que $h \in G$ divise $g \in G$ à gauche et que $k \in G$ divise $g$ à droite si $g=h k$ avec $l_{R}(g)=l_{R}(h)+l_{R}(k)$. On dit qu'un élément est équilibré si ses diviseurs à droite et à gauche sont les mêmes.

Définition 3.1. Soit $(G, R)$ un groupe positivement engendré, soit $c \in G$ un élément équilibré et soit $P_{c}$ l'ensemble des diviseurs (à gauche ou à droite) de $c$. On considère un ensemble $\underline{P}_{c}=\left\{\underline{w} \mid w \in P_{c}\right\}$ en bijection avec $P_{c}$ et on définit un monoïde noté $M\left(P_{c}\right)$ par la présentation suivante : l'ensemble des générateurs est $\underline{P}_{c}$ et les relations sont $\underline{w} \cdot \underline{w}^{\prime}=\underline{w} w^{\prime}$ pour tous les couples $\left(w, w^{\prime}\right)$ tels que $w, w^{\prime}$ et $w w^{\prime}$ sont des diviseurs de $c$ et que $l_{R}\left(w w^{\prime}\right)=l_{R}(w)+l_{R}\left(w^{\prime}\right)$.

Les notions standard de divisibilité à gauche ou à droite dans le monoïde $M\left(P_{c}\right)$ étendent les notions correspondantes de divisibilité définies dans $P_{c}$. Notons que les atomes, i.e., les éléments différents de 1 qui ne sont pas produit de deux facteurs différents de 1 , sont des éléments de $P_{c}$.

Remarquons qu'on a un morphisme de monoïdes $M\left(P_{c}\right) \rightarrow G$ donné par $\underline{w} \mapsto w$ pour $w \in P_{c}$. L'existence de ce morphisme permet facilement de prouver que $M\left(P_{c}\right)$ a une propriété de simplifiabilité partielle à gauche et à droite $(c f .[\mathrm{B}, 0.4 .4])$ : si $a m=b m$ ou si $m a=m b$ avec $a$ et $b$ dans $P_{c}$ et $m \in M\left(P_{c}\right)$ alors $a=b$. On a de plus :

Proposition 3.2. Pour tout $w \in P_{c}$ l'élément $w^{\prime}=c w c^{-1}$ est dans $P_{c}$ et est l'unique élément de $P_{c}$ tel que $\underline{w^{\prime}} \cdot \underline{c}=\underline{c} \cdot \underline{w}$. . L'application $\underline{w} \mapsto \underline{w^{\prime}}$ définit un automorphisme du monoïde $M\left(P_{c}\right)$.

On appellera "conjugaison par $\underline{c}$ " l'automorphisme ainsi défini.

Preuve. On a $c=x w$ avec $l_{R}(c)=l_{R}(w)+l_{R}(x)$. Comme $c$ est équilibré, l'élément $x$ est aussi un diviseur de $c$ à droite, donc on peut écrire $c=w^{\prime} x$ avec $l_{R}\left(w^{\prime}\right)+$ $l_{R}(x)=l_{R}(c)$. On a $w^{\prime}=c w c^{-1}$ et $\underline{c}=\underline{w}^{\prime} \cdot \underline{x}=\underline{x} \cdot \underline{w}$, d'où $\underline{c} \cdot \underline{w}=\underline{w^{\prime}} \cdot \underline{x} \cdot \underline{w}=\underline{w^{\prime}} \cdot \underline{c}$. L'application $\underline{w} \mapsto \underline{w}^{\prime}$ définit un morphisme de monoïdes. On a un morphisme en sens inverse en faisant un raisonnement analogue en partant de $w^{\prime}$. D'où le résultat. 
Notons encore la propriété générale suivante de $M\left(P_{c}\right)$ :

Proposition 3.3. Tout élément de $M\left(P_{c}\right)$ divise une puissance suffisamment grande de c.

Preuve. Tout élément de $M\left(P_{c}\right)$ s'écrit $\underline{w}_{1} \ldots \underline{w}_{k}$ pour un certain $k$, où les $w_{i}$ sont des éléments de $P_{c}$. On montre par récurrence sur $k$ que $\underline{w}_{1} \ldots \underline{w}_{k}$ divise $\underline{c}^{k}:$ par définition les éléments de $\underline{P}_{c}$ divisent $\underline{c}$, donc il existe $x \in P_{c}$ tel que $\underline{w}_{k} \underline{x}=\underline{c}$. Par 3.2 on a $\underline{w}_{1} \ldots \underline{w}_{k} \underline{x}=\underline{c}_{1}^{\prime} \ldots \underline{w}_{k-1}^{\prime}$ où les $w_{i}^{\prime}$ sont des éléments de $P_{c}$. Par hypothèse de récurrence $\underline{w}_{1}^{\prime} \ldots \underline{w}_{k-1}^{\prime}$ divise $\underline{c}^{k-1}$, d'où la proposition.

Remarquons que par définition la longueur $l_{R}$ s'étend en une fonction additive sur $M\left(P_{c}\right)$. Remarquons aussi que les diviseurs de $\underline{c}$ dans $M\left(P_{c}\right)$ sont exactement les éléments de $\underline{P}_{c}$. Les éléments $\underline{r} \in \underline{P}_{c}$ tels que $r \in R$ sont les atomes du monoïde $M\left(P_{c}\right)$.

Revenons à la situation du groupe de Coxeter $W$ de type $\tilde{A}_{n-1}$ et appliquons les constructions précédentes à un élément de Coxeter $c$ fixé de $W$ comme dans la section précédente, dont on garde les notations.

Nous prouvons maintenant dans ce cas la conjecture 1.1 (ii) :

Proposition 3.4. Le monö̈de $M\left(P_{c}\right)$ est engendré par les $\underline{r}$ où $r$ est une réflexion qui divise c avec comme relations

$$
\underline{r} . \underline{t}=\underline{r t r} . \underline{r}
$$

si $r$ et $t$ sont deux réflexions distinctes telles que rt divise $c$.

Remarquons qu'un cas particulier de ces relations est que $\underline{r} \cdot \underline{t}=\underline{t} . \underline{r}$ si $r t$ divise $c$ et que $r$ et $t$ commutent.

Preuve. La preuve suit les mêmes grandes lignes que celle de [B, 2.1.4]. Le monoïde $M\left(P_{c}\right)$ est engendré par les $\underline{r}$ où $r$ est une réflexion qui divise $c$ et les relations 3.5 sont vraies dans $M\left(P_{c}\right)$. Il suffit de voir que ces relations impliquent les autres, c'est-à-dire que pour tout $w \in P_{c}$ on peut passer d'une écriture de $w$ de longueur $l_{R}(w)$ à une autre uniquement par les relations

$$
r . s=(r s r) . r
$$

si $r$ et $s$ sont deux réflexions distinctes telles que $r s$ divise $c$. Prouvons ceci par récurrence $\operatorname{sur} l_{R}(w)$. Si $l_{R}(w)=1$ il n'y a qu'une écriture de longueur minimale de $w$. Dans le cas général il suffit de prouver que si $t \in R$ et $t \preccurlyeq w \in P_{c}$ alors à partir d'une écriture minimale de $w$ fixée et par application des relations 3.6 on peut obtenir une écriture minimale de $w$ commençant par $t$. L'hypothèse de récurrence permet alors de conclure. L'élément $w$, divisant $c$, est un produit de diviseurs élémentaires 
comme dans 2.19. On fixe une écriture minimale de $w$ obtenue par concaténation d'une écriture minimale de chaque diviseur élémentaire. Par 2.20 une réflexion $t$ divise $w$ si et seulement si elle divise un des diviseurs élémentaires de $w$. Comme deux réflexions qui interviennent dans les écritures de deux diviseurs élémentaires de $w$ distincts commutent entre elles, $t$ commute avec les réflexions qui interviennent dans les écritures minimales des autres diviseurs élémentaires. On est donc ramené à montrer le résultat pour un seul diviseur élémentaire. Si ce diviseur est un élément de Coxeter d'un groupe de type $A$ (c'est-à-dire a toutes ses orbites finies) le résultat est connu ( $c f$. . $[\mathrm{B}]$ et $[\mathrm{BDM}])$. On est ramené au cas d'un élément de Coxeter d'un groupe de type $\tilde{A}$. Il suffit donc de prouver le résultat pour $c$ lui-même. Quitte à faire une permutation circulaire des réflexions élémentaires, ce qui revient à une conjugaison donc laisse invariantes les relations 3.6, on peut ramener $c$ à être de la forme ( $c f$. preuve de 2.10)

$$
c=\left(s_{k_{h}} s_{k_{h}+1} \ldots s_{k_{h+1}-1}\right)\left(s_{k_{h-1}} s_{k_{h-1}+1} \ldots s_{k_{h}-1}\right) \ldots\left(s_{1} s_{2} \ldots s_{k_{1}-1}\right),
$$

avec $1=k_{0}<k_{1}<k_{2}<\cdots<k_{h}<k_{h+1}=n+1$ et $s_{i}=(i, i+1)$. On part de cette écriture et on veut faire apparaitre $t$ à gauche de $c$ par application des relations 3.6. En fait il suffit de faire apparaître $t$ dans une écriture de $c$; on peut ensuite le ramener à gauche par application des relations 3.6. Chaque $\left(s_{k_{i}} s_{k_{i}+1} \ldots s_{k_{i+1}-1}\right)$ est un élément de Coxeter d'un groupe de type $A$, donc on peut faire apparaître à gauche ou à droite, par application des relations 3.6 n'importe quelle réflexion de support inclus dans $\left[k_{i}, k_{i+1}\right]$. On en déduit que si $k_{i-1} \leq a \leq k_{i} \leq k_{j} \leq$ $b \leq k_{j+1}$, on peut faire apparaître dans l'écriture de $c$ par application de 3.6 le produit $\left(k_{j}, b\right)\left(k_{j-1}, k_{j}\right) \ldots\left(k_{i+1}, k_{i}\right)\left(k_{i}, a\right)$ qui est l'écriture d'un élément de Coxeter d'un groupe de type $A$, donc on peut faire apparaitre $(a, b)$ dans l'écriture de cet élément. Le même type d'argument montre qu'on peut faire apparaître $(b, a+n)$ sous les mêmes hypothèses, en faisant apparaitre le produit $(b, 1+n)$ à droite du produit $\left(s_{k_{h}} \ldots s_{k_{h+1}}\right) \ldots\left(s_{k_{j}} \ldots s_{k_{j+1}}\right)$ et $(1+n, a+n)=(1, a)$ à gauche du produit $\left(s_{k_{i}} \ldots s_{k_{i+1}-1}\right) \ldots\left(s_{k_{0}} \ldots s_{k_{1}-1}\right)$.

Il reste à voir qu'on peut faire apparaître toute réflexion $t=(a, \alpha)$ où $a \in X$ et $\alpha \in \Xi$. Comme $c$ est la translation d'une position dans le sens croissant de $X$ et d'une position dans le sens décroissant de $\Xi$, il conjugue $(a, \alpha)$ sur $\left(a^{\prime}, \alpha^{\prime}\right)$ où $a^{\prime}$ est translaté de $a$ dans $X$ d'une position dans le sens croissant et $\alpha^{\prime}$ est translaté de $\alpha$ dans $\Xi$ d'une position dans le sens décroissant. Donc $(a, \alpha)$ peut être ramené par conjugaison par une puissance de $c$ sur une réflexion d'une des formes $(a, b)$ ou $(a, b-n)$, avec $1 \leq a, b \leq n$, et par la première partie de cette démonstration on sait qu'on peut faire apparaître une telle réflexion dans une écriture de $c$ par application des relations 3.6. La conjugaison par $c$ est aussi une suite d'applications de 3.6, d'où le résultat. 


\section{Présentations duales pour les groupes d'Artin-Tits affines de type $\tilde{A}$}

Nous gardons les notations des deux sections précédentes, en particulier $c$ est un élément de Coxeter du groupe de Coxeter $W$ de type $\tilde{A}_{n-1}$. Le premier but de cette section est de prouver le théorème suivant ( $c f$. conjecture 1.1 (i)) :

Théorème 4.1. Le groupe de fractions $G\left(P_{c}\right)$ de $M\left(P_{c}\right)$ est isomorphe au groupe d'Artin-Tits de type $\tilde{A}_{n-1}$.

Nous noterons $B\left(\tilde{A}_{n-1}\right)$ le groupe d'Artin-Tits de type $\tilde{A}_{n-1}$. Il est engendré par $\boldsymbol{s}_{1}, \boldsymbol{s}_{2}, \ldots, \boldsymbol{s}_{n}$, avec comme relations $\boldsymbol{s}_{i} \boldsymbol{s}_{i+1} \boldsymbol{s}_{i}=\boldsymbol{s}_{i+1} \boldsymbol{s}_{i} \boldsymbol{s}_{i+1}$ pour $i=1, \ldots, n$, si on pose $\boldsymbol{s}_{n+1}=\boldsymbol{s}_{1}$, et $\boldsymbol{s}_{i} \boldsymbol{s}_{j}=\boldsymbol{s}_{j} \boldsymbol{s}_{i}$ si $i \neq j \pm 1(\bmod n)$. Pour prouver le théorème 4.1 on montre d'abord que dans $G\left(P_{c}\right)$ les éléments $\underline{s}_{1}, \ldots, \underline{s}_{n}$ vérifient les mêmes relations de tresses. Ceci définit un morphisme $B\left(\tilde{A}_{n-1}\right) \rightarrow G\left(P_{c}\right)$. On trouve ensuite dans $B\left(\tilde{A}_{n-1}\right)$ des éléments dont les images par ce morphisme sont les générateurs $\underline{r}$ de $G\left(P_{c}\right)$ et qui vérifient les relations 3.5 , ce qui prouve la bijectivité du morphisme. Pour cette deuxième étape on utilisera l'interprétation de $B\left(\tilde{A}_{n-1}\right)$ comme groupe fondamental.

Proposition 4.2. L'application $s_{i} \mapsto \underline{s}_{i}$ pour $i=1, \ldots, n$ se prolonge en un homomorphisme $B\left(\tilde{A}_{n-1}\right) \rightarrow G\left(P_{c}\right)$.

Preuve. Cela revient à montrer que dans $G\left(P_{c}\right)$ on a $\underline{s}_{i} \underline{s}_{i+1} \underline{s}_{i}=\underline{s}_{i+1} \underline{s}_{i} \underline{s}_{i+1}$ pour $i=1, \ldots, n$ et $\underline{s}_{i} \underline{s}_{j}=\underline{s}_{j} \underline{s}_{i}$ si $i, j \in[1, n]$ et $|i-j| \geq 2$. Par 3.4 on a $\underline{s}_{i} \underline{s}_{i+1}=$ $\underline{s}^{\prime} \underline{s}_{i}=\underline{s}_{i+1} \underline{s}^{\prime}$, où $s^{\prime}=(i, i+2)$. On en déduit $\underline{s}_{i+1} \underline{s}_{i} \underline{s}_{i+1}=\underline{s}_{i+1} \underline{s}^{\prime} \underline{s}_{i}=\underline{s}_{i} \underline{s}_{i+1} \underline{s}_{i}$. On a aussi par $3.4 \underline{s}_{i} \underline{s}_{j}=\underline{s}_{j} \underline{s}_{i}$ si $|i-j| \geq 2$.

Avant de montrer que ce morphisme est un isomorphisme, nous rappelons l'interprétation de $B\left(\tilde{A}_{n-1}\right)$ comme sous-groupe du groupe de tresses à $n$ brins dans $\mathbb{C}^{*}$ $(c f$. [GL] et $[\mathrm{A}])$. On considère un $n$-uplet de points de $\mathbb{C}^{*}$. Le groupe des tresses dans $\mathbb{C}^{*}$ de base ce $n$-uplet ("tresses à $n$ brins" dans $\mathbb{C}^{*}$ ), est isomorphe au groupe d'Artin-Tits $B\left(B_{n}\right)$ de type $B_{n}$. L'application qui associe à une telle tresse le nombre de tours total des brins autour de 0 est un morphisme à valeur dans $\mathbb{Z}$. Le groupe des tresses de type $\tilde{A}_{n-1}$ est le noyau de ce morphisme. Notons aussi qu'on peut considérer le groupe des tresses à $n$ brins dans $\mathbb{C}^{*}$ de base le $n$-uplet $\left(x_{1}, \ldots, x_{n}\right)$ comme le sous-groupe du groupe des tresses à $n+1$ brins dans $\mathbb{C}$ de base le $n+1$-uplet $\left(0, x_{1}, \ldots, x_{n}\right)$ tel que le brin issu de l'origine soit trivial (tresses pures relativement à un brin fixé).

Nous nous plaçons dans le cadre de 2.15 et nous choisissons comme $n$-uplet de base $(a, b, \ldots, l, \alpha, \ldots, \lambda)$ comme dans 2.15 . Nous associons à chaque $\underline{r}$ où $r$ est une réflexion de $P_{c}$ une tresse de $B\left(\tilde{A}_{n-1}\right)$ la façon suivante $: r$ est représentée par un chemin $\gamma$ dans la couronne, sans auto-intersection, reliant $i$ à $j$ où $i$ et $j$ sont les 
images dans la couronne de deux points de $X \cup \Xi$. On associe à ce chemin la tresse où tous les points sont fixes sauf les deux points partant respectivement de $i$ et $j$ qui suivent $\gamma$ en sens inverse et s" "évitent par la droite" si l'orientation du plan est choisie dans le sens horaire ce que nous supposerons dans les figures qui suivent. Plus précisément, on peut supposer que $\gamma$ est une application différentiable de $[0,1]$ dans la couronne telle que les tangentes en 0 et 1 soient orthogonales au bord de la couronne. Soit $\vec{n}(t)$ un vecteur normal dans le sens direct à $\gamma$ en $\gamma(t)$. On considère la tresse où tous les brins sont fixes sauf un brin partant de $i$ donné par $t \mapsto \gamma(t)+\varepsilon \sin (\pi t) \vec{n}(t)$ et un brin partant de $j$ donné par $t \mapsto \gamma(1-t)-\varepsilon \sin (\pi(1-t)) \vec{n}(1-t)$ où $\varepsilon$ est assez petit pour que la tresse soit dans la couronne.

Proposition 4.3. L'application que nous venons de définir se prolonge en un isomorphisme de $G\left(P_{c}\right)$ dans $B\left(\tilde{A}_{n-1}\right)$ inverse de l'homomorphisme défini par 4.2.

Preuve. Il faut voir que les relations 3.5 sont vérifiées par les images des éléments $\underline{r}$. On déduit de 2.19 qu'il y a trois types de couples $(r, t) \in R^{2}$ tels que $r t$ divise $c$.

- Si $r$ et $t$ correspondent à des chemins sans intersection $\underline{r}$ et $\underline{t}$ commutent et il est clair que leurs images dans $B\left(\tilde{A}_{n-1}\right)$ commutent aussi.

- Si $r=(i, j)$ et $t=(j, k)$ où $i, j$ et $k$ sont deux à deux distincts modulo $n$ et si $i, j, k$ sont les sommets d'un triangle curviligne direct dans la bande, la relation est $(i, j) .(j, k)=(i, k) .(i, j)$. Cette relation est vérifiée par les tresses images : si nous notons encore $i, j$ et $k$ les images respectives dans la couronne des points $i, j$ et $k$, les éléments $(i, j),(j, k)$ et $(i, k)$ correspondent à des chemins

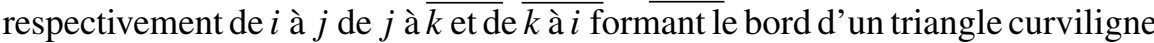
direct inclus dans la couronne et la relation pour les tresses correspondantes n'est autre que la relation classique pour les tresses à trois brins (voir figure 3) dans le groupe de tresses de $\mathbb{C}^{*}$ (ou de $\mathbb{C}$ ).

- Si $r=(x, \xi)$ et $t=(x-n, \xi)$ avec $x \in X$ et $\xi \in \Xi$, la relation est $(x, \xi) \cdot(x-n, \xi)=(x+n, \xi) .(x, \xi)$. La relation correspondante est vraie dans

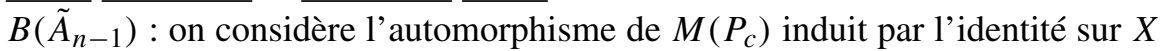
et la translation de $n$ sur $\Xi$. La relation revient à dire que $(x, \xi) .(x-n, \xi)$ est invariant par cet automorphisme. Dans $B\left(\tilde{A}_{n-1}\right)$ on considère l'automorphisme induit par une isotopie qui est l'identité sur le bord extérieur de la couronne et fait tourner l'autre bord de la couronne d'un tour dans le sens positif. L'application de l'énoncé est compatible avec ces automorphismes. On peut supposer $x \in[1, n]$; en appliquant une puissance convenable de ces deux automorphismes on peut ramener $\xi$ dans $[1, n]$; la relation résulte alors de la figure 4 qui permet de voir l'invariance cherchée.

Les propositions 4.2 et 4.3 prouvent le théorème 4.1 . 

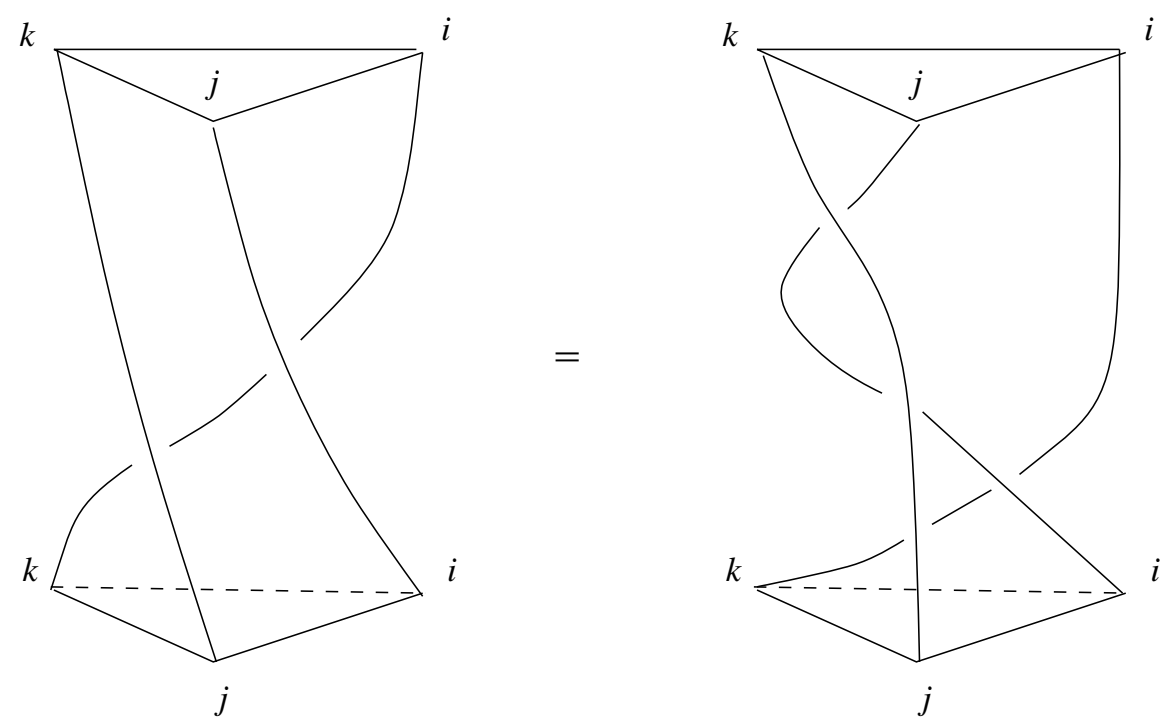

Figure 3. $\underline{(i, j)} . \underline{(j, k)}=\underline{(j, k)} . \underline{(i, k)}$.

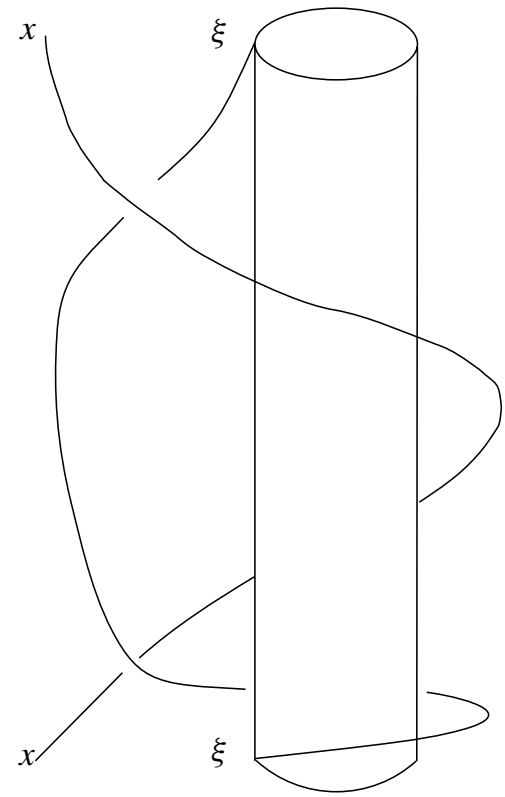

Figure 4. $\underline{(x, \xi)} \cdot \underline{(x, \xi+n)}$. 
Nous avons ainsi défini pour chaque choix d'un élément de Coxeter $c$ un sousmonoïde $M\left(P_{c}\right)$ de $B\left(\tilde{A}_{n-1}\right)$. Deux tels monoïdes correspondant à des ensembles $\{a, \ldots, l\}$ et $\{\alpha, \ldots, \lambda\}$ de mêmes cardinaux respectifs sont isomorphes. L'échange de $X$ et de $\Xi$ est aussi un isomorphisme (qui se traduit dans la représentation géométrique par une rotation de $\pi$ de la bande). On en déduit que la classe d'isomorphisme de $M\left(P_{c}\right)$ ne dépend que de la partition de $n$ en $|X \cap[1, n]|+|\Xi \cap[1, n]|$. Réciproquement :

Théorème 4.4. L'application qui à c associe l'ensemble $\{|X \cap[1, n]|,|\Xi \cap[1, n]|\}$ induit une bijection des classes d'isomorphisme des monoüdes $M\left(P_{c}\right)$ de la section 3 sur les partitions de $n$ en deux parties.

Ce théorème résulte des considérations qui précèdent et de la proposition suivante qui montre que $|X \cap[1, n]|$ et $|\Xi \cap[1, n]|$ sont déterminés par l'action de la conjugaison $\operatorname{par} \underline{c}$ sur les atomes.

Proposition 4.5. L'orbite par la conjugaison par $\underline{c}$ d'un atome $(i, j)$ est finie de cardinal $|X \cap[1, n]|$ (resp. $|\Xi \cap[1, n]|)$ si $i$ et $j$ sont tous deux dans $X$ (resp. tous deux dans $\Xi$ ) et infinie si $i \in X$ et $j \in \Xi$.

Preuve. Par 3.2, si $r$ et $r^{\prime}$ sont des réflexions de $P_{c}$ on a $\underline{r} c=\underline{c} \underline{r}^{\prime}$ si et seulement si $r c=c r^{\prime}$. On a vu dans la démonstration de 3.4 que la conjugaison par $c$ revient à décaler $X$ d'une position dans le sens croissant et $\Xi$ d'une position dans le sens décroissant. On en déduit le résultat.

\section{Une structure à la Garside pour les groupes d'Artin-Tits affines de type $\tilde{A}$}

Gardons les notations des sections précédentes. Nous allons étudier les propriétés de la divisibilité dans $P_{c}$ dans le cas où $c=s_{1} s_{2} \ldots s_{n}$. Dans ce cas on a $c=$ $(2,3, \ldots, n)_{[1]}(1)_{[-1]}$ et les ensembles $\Xi$ et $X$ sont respectivement $\Xi=\{z \in \mathbb{Z} \mid$ $z \equiv 1(\bmod n)\}$ et $X=\{z \in \mathbb{Z} \mid z \not \equiv 1(\bmod n)\}$.

La propriété fondamentale du monoïde $M\left(P_{c}\right)$ dans ce cas (théorème 5.4) est une conséquence de la proposition suivante :

Proposition 5.1. Si $c=s_{1} \ldots s_{n}$, deux atomes quelconques de $P_{c}$ ont un ppcm dans $P_{c}$.

Preuve. Considérons deux atomes $r=(x, y)$ et $r^{\prime}=\left(x^{\prime}, y^{\prime}\right)$. On sait que $r$ (resp. $r^{\prime}$ ) divise un élément $p \in P_{c}$ si et seulement si $x$ et $y$ (resp. $x^{\prime}$ et $y^{\prime}$ ) sont dans la même partie de la partition associée à $p$ comme dans 2.22. Il y a deux cas. 
- Si $r$ et $r^{\prime}$ sont sans croisement, ils commutent et $r r^{\prime}$ est dans $P_{c}$ par 2.19. Dans la décomposition d'un multiple commun $m$ de $r$ et $r^{\prime}$ en produit de diviseurs élémentaires, ou bien $r$ et $r^{\prime}$ divisent deux diviseurs élémentaires de $m$ différents et $r r^{\prime}$ divise alors $m$, ou bien $r$ et $r^{\prime}$ divisent le même diviseur élémentaire $c^{\prime}$ de $m$ qui est un élément de Coxeter d'un sous-groupe quasi-parabolique $W^{\prime}$ et on conclut que $r r^{\prime}$ divise $c^{\prime}$ soit par 2.19 si $W^{\prime}$ est de type $\tilde{A}$ soit par [BDM, 1.8] si $W^{\prime}$ est de type $A$. Donc le ppcm de $r$ et $r^{\prime}$ existe et vaut $r r^{\prime}$.

- Si $r$ et $r^{\prime}$ se croisent (i.e. ne sont pas sans croisement), supposons $x<y, x^{\prime}<y^{\prime}$ et $x$ et $x^{\prime}$ dans $[1, n]$; si $m$ est un multiple commun de $r$ et $r^{\prime}$, alors $\{x, y\}$ et $\left\{x^{\prime}, y^{\prime}\right\}$ ne peuvent pas être dans deux parties distinctes de la partition associée à $m$ car ces deux parties ne seraient pas sans croisement. Donc il existe un diviseur élémentaire $m^{\prime}$ qui divise $m$ et qui est multiple de $r$ et $r^{\prime}$. Distinguons trois cas.

Si l'ensemble $\left\{x, y, x^{\prime}, y^{\prime}\right\} \cap X$ est inclus dans un intervalle de longueur strictement inférieure à $n$, la partition dont les seules parties non triviales sont $\left\{x, y, x^{\prime}, y^{\prime}\right\}+k n$ avec $k \in \mathbb{Z}$ définit un élément de Coxeter $c^{\prime}$ d'un quasiparabolique de type $A$.

Si l'ensemble $\left\{x, y, x^{\prime}, y^{\prime}\right\} \cap X$ n'est pas inclus dans un intervalle de longueur strictement inférieure à $n$ et si $\left\{x, y, x^{\prime}, y^{\prime}\right\} \cap \Xi$ est non vide, la partition dont la seule partie non triviale est $\left\{x, y, x^{\prime}, y^{\prime}\right\}+n \mathbb{Z}$ définit un élément de Coxeter $c^{\prime}$ d'un quasi-parabolique de type $\tilde{A}$.

Si l'ensemble $\left\{x, y, x^{\prime}, y^{\prime}\right\} \cap X$ n'est pas inclus dans un intervalle de longueur strictement inférieure à $n$ et si $\left\{x, y, x^{\prime}, y^{\prime}\right\} \cap \Xi$ est vide, notons $c^{\prime}$ l'élément de Coxeter d'un quasi-parabolique de type $\tilde{A}$ défini par la partition dont la seule partie non triviale est $\left\{1, x, y, x^{\prime}, y^{\prime}\right\}+n \mathbb{Z}$ et remarquons que la partie associée à $m^{\prime}$ étant infinie doit contenir 1 .

Dans les trois cas l'élément $c^{\prime}$ divise $m^{\prime}$ par 2.19 appliqué à $m^{\prime}$ et il est multiple de $r$ et $r^{\prime}$. C'est donc le ppcm de $r$ et $r^{\prime}$ dans $P_{c}$.

On peut alors appliquer la généralisation immédiate suivante de [B, 0.5.2] :

Théorème 5.2. Soit $(G, R)$ un groupe positivement engendré, soit c un élément équilibré et soit $M\left(P_{c}\right)$ comme précédemment. Supposons que deux éléments quelconques de $R$ ont un ppcm dans $P_{c}$; alors la divisibilité à gauche et la divisibilité à droite donnent à $M\left(P_{c}\right)$ deux structures de treillis.

Dans ce contexte on peut vérifier que les résultats de [BDM, section 2] et [B, 0.5] s'appliquent.

Nous appellerons structure quasi-Garside une structure de monoïde vérifiant tous les axiomes de [B, 0.5.1] sauf la finitude du nombre d'atomes. Nos axiomes seront donc : 
Définition 5.3. Un monoïde $M$ est dit quasi-Garside si

(i) Pour tout $m \in M$ le nombre de facteurs dans un produit égal à $m$ est borné.

(ii) $M$ est simplifiable à gauche et à droite.

(iii) La divisibilité à gauche et la divisibilité à droite donnent à $M$ deux structures de treillis.

(iv) Il existe un élément $\Delta$ (élément de Garside) dont l'ensemble des diviseurs à gauche est égal à l'ensemble des diviseurs à droite et engendre $M$.

L'axiome (i) signifie que le monoïde est atomique au sens par exemple de [B, $0.2 .2]$; il est équivalent à l' existence d'une longueur $l$ sur le monoïde telle que $l(a b) \geq$ $l(a)+l(b)$ pour tout couple $(a, b)$.

Les axiomes (i), (ii) et (iv) étant vérifiés par un monoïde défini comme plus haut à partir d'un groupe engendré et d'un élément équilibré, l'énoncé 5.2 devient :

Théorème 5.4. Sous les hypothèses de 5.2, le monö̈de $M\left(P_{c}\right)$ est un monoüde quasiGarside avec c comme élément de Garside.

Ce théorème s'applique en particulier à un élément $c$ comme dans 5.1. Un tel choix définit donc une structure quasi-Garside sur le groupe d'Artin-Tits de type $\tilde{A}_{n-1}$.

Le résultat suivant montre que le choix de $c$ fait dans 5.1 est à isomorphisme près le seul pour lequel la divisibilité a une structure de treillis.

Proposition 5.5. Si c est un élément de Coxeter d'un groupe de type $\tilde{A}_{n-1}$, le monö̈de $M\left(P_{c}\right)$ muni de l'ordre de la divisibilité a une structure de treillis (et est donc un monoïde quasi-Garside) si et seulement si l'un des deux ensembles $\Xi$ ou X est réduit à un seul élément modulo $n$.

Preuve. L'échange de $\Xi$ et $X$ définit un isomorphisme des monoïdes correspondants. D'autre part deux éléments de Coxeter tels que les ensembles $X$ correspondants ont même nombre d'éléments modulo $n$ sont conjugués, donc dans ce cas aussi les monoïdes sont isomorphes. On en déduit par 5.1 que si $\Xi$ ou $X$ a un seul élément modulo $n$, on a bien une structure de treillis. Inversement supposons que modulo $n$, à la fois $\Xi$ et $X$ ont au moins deux éléments. Soient $a<b$ dans $X \cap[1, n]$ (resp. $\alpha \neq \beta$ dans $\Xi \cap[1, n])$. Les éléments $(a, b)$ et $(b, a+n)$ divisent $(a, b)_{[1]}(\alpha)_{[-1]}$ et $(a, b)_{[1]}(\beta)_{[-1]}$ qui sont de longueur 3 et n'ont aucun diviseur commun de longueur 2 d'après 2.19. Donc $(a, b)$ et $(b, a+n)$ n'ont pas de ppcm.

Remarquons que dans l'exemple précédent c'est le dernier cas de la preuve de 5.1, dans lequel on a dû introduire le quasi-parabolique de type $\tilde{A}$ défini par $\left\{1, x, y, x^{\prime}, y^{\prime}\right\}+n \mathbb{Z}$, qui est en défaut. En fait les raisonnements des autres cas s'appliquent pour tout élément de Coxeter $c$ mais ce dernier cas utilise le fait que $\Xi$ est un singleton modulo $n$. 
Donnons quelques conséquences de l'existence d'une structure quasi-Garside sur $B\left(\tilde{A}_{n-1}\right)$. Ces conséquences sont de simples applications des propriétés générales des monoïdes de Garside dont on vérifie qu'elles sont encore valables dans le cadre quasiGarside. Le premier est l'existence de formes normales telles que dues à Garside (voir par exemple [M] ou [BDM, section 2], voir aussi [C]).

Proposition 5.6. (i) Tout élément de $B\left(\tilde{A}_{n-1}\right)$ s'écrit de façon unique $a^{-1} b$ où $a$ et $b$ sont des éléments de $M\left(P_{c}\right)$ premiers entre eux.

(ii) Tout élément de $M\left(P_{c}\right)$ s'écrit de façon unique $a_{1} a_{2} \ldots a_{k}$ où pour $i=1, \ldots, k$ l'élément $a_{i} \in \underline{P}_{c}$ est un (le) diviseur maximal dans $\underline{P}_{c}$ du produit $a_{i} a_{i+1} \ldots a_{k}$ et $a_{k} \neq 1$.

Nous prouvons maintenant :

Proposition 5.7. Le centre de $B\left(\tilde{A}_{n-1}\right)$ est trivial.

Preuve. La démonstration suit les mêmes idées que la démonstration classique pour les monoïdes de tresses ou que la démonstration de [P, 4.1]; ces démonstrations ne s'appliquent pas telles quelles car elles supposent qu'il y a un nombre fini d'atomes. On utilise le lemme suivant :

Lemme 5.8. Soit $M$ un monoüde quasi-Garside et soit b un élément quasi-central de $M, c$ 'est-à-dire tel qu'il existe un automorphisme $\tau$ de $M$ vérifiant $x b=b \tau(x)$ pour tout $x \in M$; soit $x$ un diviseur à gauche de $b$ et $y \in M$; posons $\operatorname{ppcm}(x, y)=y z$ avec $z \in M$ : alors $z$ divise $b$ à gauche.

Preuve. Comme $x$ divise $b$ il divise $b \tau(y)=y b$, donc $y z$ divise $y b$ et par simplifiabilité $z$ divise $b$.

On en déduit la proposition : Soit $g \in B\left(\tilde{A}_{n-1}\right)$ central. On peut écrire $g=\underline{c}^{n} b$ avec $b \in M\left(P_{c}\right)$ non divisible par $\underline{c}$ et $n \in \mathbb{Z}$ convenable. On a alors $b$ quasi-central. Montrons par l'absurde que $b=1$. Sinon, soit $r$ une réflexion de $P_{c}$ telle que $\underline{r}$ divise $b$. Pour tout triplet de réflexions $(r, s, t)$ correspondant à un triangle direct comme dans la preuve de 4.3 on a $\underline{r} \cdot \underline{s}=\underline{s} \cdot \underline{t}=\underline{t} \cdot \underline{r}=\operatorname{ppcm}(\underline{r}, \underline{s})=\operatorname{ppcm}(\underline{s}, \underline{t})$. On en déduit par le lemme que $\underline{r}$ et $\underline{t}$ divisent $b$. Comme à partir de $r$, de proche en proche on peut faire apparaitre n'importe quelle réflexion $r^{\prime}$ de $P_{c}$ dans un triangle direct, on en déduit que $b$ est multiple de tous les atomes de $M\left(P_{c}\right)$ donc est multiple de $\underline{c}$, ce qui est contradictoire. Tout élément central est donc une puissance de $\underline{c}$. Or la conjugaison $\operatorname{par} \underline{c}$ est un automorphisme d'ordre infini ( $c f .3 .4$ ou 4.5), donc aucune puissance de $\underline{c}$ autre que $\underline{c}^{0}$ n'est centrale.

Donnons une dernière conséquence de l'existence de la structure quasi-Garside. 
Proposition 5.9. Soient $c=s_{1} \ldots s_{n}$ et $\underline{c}=\underline{s}_{1} \ldots \underline{s}_{n}$ comme précédemment. Le centralisateur de $\underline{c}^{h}$ dans le groupe d'Artin-Tits de type $\tilde{A}_{n-1}$ engendrépar $\underline{s}_{1}, \ldots, \underline{s}_{n}$ est un groupe d'Artin-Tits de type $B_{\operatorname{pgcd}(h, n-1)}$.

Preuve. La théorie générale des monoïdes de (quasi-)Garside énonce que les points fixes d'un automorphisme $\sigma$ dans un monoïde $M$ de (quasi-)Garside forment un monoïde $M^{\sigma}$ de (quasi-)Garside, avec pour atomes certains des ppcm des orbites des atomes et même élément de Garside; le groupe des fractions de ce monoïde est le groupe des points fixes de $\sigma$ dans le groupe des fractions de $M$ (cf. [BDM, 2.26] dont la démonstration s'étend à la situation quasi-Garside). Nous appliquons ceci à la conjugaison par $\underline{c}^{h}$. Le centralisateur de $\underline{c}^{h}$ dans $G\left(P_{c}\right)$ est donc engendré par les ppcm des orbites des atomes sous la conjugaison par $c^{h}$. Comme le montre le calcul fait dans 3.4 la conjugaison $\operatorname{par} \underline{c}$ envoie $(x, y) \operatorname{sur}\left(x^{\prime}, y^{\prime}\right)$ où $x^{\prime}$ et $y^{\prime}$ s'obtiennent à partir de $x$ et de $y$ en translatant $X$ d'une position dans le sens croissant et $\Xi$ d'une position dans le sens décroissant. Pour simplifier au lieu d'indexer comme précédemment les éléments de $X$ par les entiers non congrus à 1 modulo $n$, nous renumérotons consécutivement les éléments de $X$, en les notant $x_{i}$ avec $i \in \mathbb{Z}$, et nous faisons de même pour les éléments de $\Xi$ qui seront notés $\xi_{i}$ avec $i \in \mathbb{Z}$. La translation de $n$ devient alors $x_{i} \mapsto x_{i+n-1}$ et $\xi_{i} \mapsto \xi_{i+1}$. La conjugaison par $\underline{c}^{h}$ envoie $\left(x_{i}, x_{j}\right)$ sur $\left(x_{i+h}, x_{j+h}\right)$. L'orbite de $\left(x_{i}, x_{j}\right)$ ne dépend donc que du pgcd de $h$ et de $\overline{n-1 \text {. La conjugaison }}$ ar $\underline{c}^{h}$ envoie $\overline{\left(\xi_{0}, x_{j}\right)}$ sur $\left(\xi_{0}, x_{j+n h}\right)$. Le ppcm d'une telle orbite est $\left(\xi_{0}\right)_{[-1]}\left(x_{j}, x_{j+k}, x_{j+2 k}, \ldots, \overline{\left.x_{(j+n-1}-k\right)}\right)_{[1]}$ où $k$ est le pgcd de $h$ et $n-1$. On voit que les ppcm des orbites d'atomes ne dépendent que du pgcd de $n-1$ et $h$. Donc $\underline{c}^{h}$ et $\underline{c}^{\operatorname{pgcd}(n-1, h)}$ ont même centralisateur. On est donc ramené au cas où $h$ divise $n-1$. Le lemme suivant est le cas particulier de la proposition quand $h=n-1$.

Lemme 5.10. Le centralisateur de $\underline{c}^{n-1}$ est un groupe d'Artin-Tits de type $B_{n-1}$ et l'image de $\underline{c}$ dans ce groupe est un élément de Coxeter de ce groupe.

On a appelé élément de Coxeter d'un groupe d'Artin-Tits le relevé canonique d'un élément de Coxeter du groupe de Coxeter.

Preuve. Les éléments $\left(x_{i}, x_{j}\right)$ sont centralisés par $\underline{c}^{n-1}$. L'orbite de $\left(\xi_{0}, x_{j}\right)$ se com-


$\left(\xi_{0}\right)_{[-1]}\left(x_{j}\right)_{[1]}$. Le centralisateur $C\left(\underline{c}^{n-1}\right)$ de $\underline{c}^{n-1}$ est donc engendré par ces éléments et toutes les relations s'obtiennent en égalant les décompositions de $\underline{c}$ comme produits de ces générateurs. On a

$$
\underline{c}=\underline{\left(x_{1}, x_{2}\right)} \cdot \underline{\left(x_{2}, x_{3}\right)} \cdots \underline{\left(x_{n-2}, x_{n-1}\right)} \cdot \underline{\left(\xi_{0}\right)_{[-1]}\left(x_{n-1}\right)_{[1]}} .
$$


Pour construire un isomorphisme entre $C\left(\underline{c}^{n-1}\right)$ et le groupe d'Artin-Tits $B\left(B_{n-1}\right)$ de type $B_{n-1}$ nous revenons à l'interprétation de $B\left(\tilde{A}_{n-1}\right)$ comme tresses dans une couronne. Remarquons que les générateurs ci-dessus de $C\left(c^{n-1}\right)$ sont des tresses telles que le brin d'origine $\xi_{0}$ a pour extrémité $\xi_{0}$ (voir figure 5). Or on peut interpréter le groupe de tresses de type $B_{n-1}$ comme le groupe de tresses à $n-1$ brins dans la couronne, les points de base étant les points $x_{1}, x_{2}, \ldots, x_{n-1}$ (cf., [L]). On a donc un morphisme de $C\left(\underline{c}^{n-1}\right)$ dans $B\left(B_{n-1}\right)$ par oubli du brin issu de $\xi_{0}$. Les générateurs de $C\left(\underline{c}^{n-1}\right)$ s'envoient sur les générateurs de la présentation duale du groupe $B\left(B_{n-1}\right)$ et l'image de $\underline{c}$ est l'élément de Coxeter de $B\left(B_{n-1}\right)$. Donc les relations entre les générateurs de ces deux groupes se correspondent. Le morphisme est donc un isomorphisme.

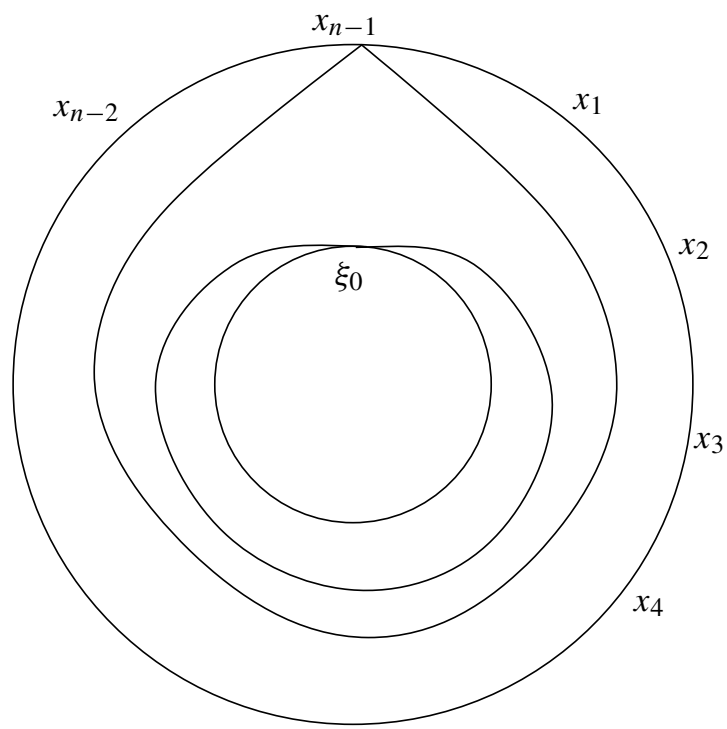

Figure 5. $\left(\xi_{0}\right)_{[-1]}\left(x_{n-1}\right)_{[1]}$.

Prouvons alors la proposition. Si $h$ divise $n-1$, le centralisateur de $\underline{c}^{h}$ est égal au centralisateur de $\underline{c}^{h}$ dans le centralisateur de $\underline{c}^{n-1}$. Le lemme permet donc de terminer la démonstration de la proposition car le centralisateur d'une puissance d'un élément de Coxeter dans un groupe de tresses de type $B$ est connu par les résultats de $[\mathrm{BDM}]$.

Remarque 5.11. On obtient des générateurs standards (i.e. vérifiant les relations de tresses de type $B)$ du centralisateur de $\underline{c}^{n-1}$ en prenant $\left(x_{1}, x_{2}\right),\left(x_{2}, x_{3}\right), \ldots$, $\left(x_{n-2}, x_{n-1}\right)$, et $\left(\xi_{0}\right)_{[-1]}\left(x_{n-1}\right)_{[1]}$. D'autre part il est facile de voir que le centralisateur de $\left(s_{1} s_{2} \ldots s_{n}\right)^{n-1}$ dans le groupe de Coxeter $W\left(\tilde{A}_{n-1}\right)$ est le groupe le 
groupe engendré par $\left(x_{1}, x_{2}\right),\left(x_{2}, x_{3}\right), \ldots,\left(x_{n-2}, x_{n-1}\right)$, et $\left(\xi_{0}\right)_{[-1]}\left(x_{n-1}\right)_{[1]}$ donc est l'image du centralisateur de $\underline{c}^{n-1}$ dans le groupe d'Artin-Tits. La présentation de cette image s'obtient en ajoutant aux relations de tresses de type $B$ le fait que les $n-2$ premiers générateurs sont d'ordre 2 (le dernier est d'ordre infini).

Remerciements. Je remercie tout particulièrement Eddy Godelle pour ses nombreuses remarques pertinentes sur une version antérieure de cet article. Je remercie également Jean Michel avec qui j'ai eu plusieurs discussions sur ces résultats.

\section{Références}

[A] D. Allcock, Braid pictures for Artin groups, Trans. Amer. Math. Soc. 354 (2002), 3455-3474. Zbl 1059.20032 MR 1911508

[B] D. Bessis, The dual braid monoid. Ann. Sci. École Norm. Sup. (4) 36 (2003), 647-683. Zbl 02128583 MR 2032983

[BDM] D. Bessis, F. Digne et J. Michel, Springer theory in braid groups and the Birman-Ko-Lee monoid. Pacific J. Math. 205 (2003), 287-309. Zbl 1056.20023 MR 1922736

[BKL] J. Birman, K. H. Ko, S. J. Lee, A new approach to the word and conjugacy problem in the braid groups. Adv. Math. 139 (1998), 322-353. Zbl 0937.20016 MR 1654165

[C] R. Charney, Geodesic automation and growth functions for Artin groups of finite type. Math. Ann. 301 (1995), 307-324. Zbl 0813.20042 MR 1314589

[D] M. J. Dyer, On minimal lengths of expressions of Coxeter group elements as product of reflections. Proc. Amer. Math. Soc. 129 (2001), 2591-2595. Zbl 0980.20028 MR 1838781

[GL] J. J. Graham et G. I. Lehrer, Algèbre de diagrammes, algèbres de Hecke et nombres de décomposition aux racines de l'unité. Ann. Sci. École Norm. Sup. (4) 36 (2003), 479-524. Zbl 1062.20003 MR 2013924

[L] S. Lambropoulou, Solid torus links and Hecke algebras of $B$-type. In Proceedings of the Conference on Quantum Topology, Manhattan, World Scientific Publishing, Singapore 1994, 225-245. Zbl 0884.57004 MR 1309934

[M] J. Michel, A note on words in braid monoids. J. Algebra 215 (1999), 366-377. Zbl 0937.20017 MR 1684142

[P] M. Picantin, The center of thin Gaussian groups. J. Algebra 245 (2001), 92-122. Zbl 1002.20022 MR 1868185

[S] J. Y. Shi, The Kazhdan-Lusztig cells in certain affine Weyl groups. Lecture Notes in Math. 1179, Springer-Verlag, Berlin 1986. Zbl 0582.20030 MR 0835214

Received September 6, 2004

F. Digne, LAMFA, Université de Picardie-Jules Verne 33, Rue Saint-Leu, 80039 Amiens

Cedex, France

E-mail: digne@u-picardie.fr 